\title{
Avaliação de floculantes em processo de sedimentação visando o tratamento de água com alto teor de sólidos
}

\author{
B. D. EUSTÁQUIO ${ }^{1}$ e K. C. S. FIGUEIREDO ${ }^{2}$ \\ ${ }^{1}$ Pontifícia Universidade Católica de Minas Gerais, Faculdade de Engenharia Química \\ ${ }^{2}$ Universidade Federal de Minas Gerais, Departamento de Engenharia Química
}

\begin{abstract}
RESUMO - Considerando a tragédia ocorrida em Mariana devido ao rompimento da barragem da Mineradora Samarco em 2015, fez-se necessário um tratamento para a água do Rio Doce. Como a unidade da mineradora conduz um processo de beneficiamento de minério, os principais contaminantes da água do Rio foram sólidos finos provenientes da lama, o que torna a floculação uma rota possível para o tratamento do mesmo. Para garantir a sustentabilidade da rota sugerida, propôs-se o uso de floculantes naturais e biodegradáveis, como a quitosana, a gelatina e o alginato, materiais de origem vegetal e de baixo custo. Foram realizados testes com os três reagentes em Jar Test, para definir o melhor floculante, bem como sua dosagem. Os testes foram realizados com uma solução sintética de água com adição de argila, que foi feita de acordo com os relatórios ambientais disponíveis. Através do teste de bancada, foi possível avaliar a redução da turbidez a partir da floculação, bem como a cinética de sedimentação. $O$ alginato foi o floculante que apresentou os melhores resultados, com redução de turbidez de 2000 para 10 NTU. Os demais reagentes também apresentaram grandes eficiências na remoção de turbidez. Em relação à cinética, foram encontrados melhores ajustes para sistemas de $2^{\mathrm{a}}$ ordem nos três casos. Concentrações muito baixas de floculantes, $3 \mathrm{mg} . \mathrm{L}^{-1}$, foram suficientes para promover um tratamento satisfatório.
\end{abstract}

\section{INTRODUÇÃO}

Os resíduos industriais gerados em processos de produção, como no beneficiamento de minério, são de grande preocupação por parte das empresas e dos órgãos socioambientais. Tal problema se deve à composição desse efluente que consiste, na maioria dos casos, em metais pesados, que são componentes altamente tóxicos, e sólidos finos, que podem prejudicar a vida aquática quando em alta concentração no meio por aumentar a turbidez da água (Kurniawan et al., 2006). Tais fatores justificam a necessidade da correta destinação de efluentes, que normalmente são levados e reservados em barragens, como foi o caso da mineradora Samarco, localizada na cidade de Mariana, em Minas Gerais, Brasil. Com o rompimento da barragem dessa mineradora, iniciou-se a busca por alternativas de tratamento de água 
contaminadas por sólidos e metais, para que o Rio Doce contaminado pudesse ser recuperado. Dentre os possíveis tratamentos, tem-se a floculação.

A floculação é um processo que tem grande potencial em tratar águas contaminadas por metais pesados e sólidos. Esse tratamento consiste basicamente no uso de um floculante que, ao entrar em contato com a água, tende a atrair os metais e outros materiais em suspensão. Sendo assim, estes se ligam ao floculante, que então se tornaria uma partícula cada vez mais densa, ocasionando a sedimentação. Assim então o processo é finalizado, tendo como resultado uma água clarificada, potencialmente com menor teor de metais pesados, turbidez reduzida e um lodo residual de fácil separação (Kurniawan et al., 2006).

Dentre os floculantes existentes, foram escolhidos os biofloculantes quitosana, gelatina e alginato de sódio, por seu baixo custo e por serem não tóxicos ao meio ambiente. (Salehizadeh e Yan, 2014). Sintetizado pela desacetilação alcalina da quitina, a quitosana tem apresentado aplicações em vários campos como na biotecnologia, biomedicina e no tratamento de água. É de fácil acesso e tem baixo custo, já que provém do segundo polímero mais abundante no mundo. É composta por uma cadeia longa, com grupos amina por todo comprimento, que podem ser protonados em meio ácido, tornando a quitosana um polímero de caráter catiônico. Essa característica faz com que esse biofloculante tenha um bom desempenho no processo de floculação, pois tem um potencial em capturar ânions presentes em suspensão por toda sua cadeia (Yang et al, 2016).

O outro biofloculante investigado para análise foi o alginato, que é um polissacarídeo natural, sendo o seu principal sal solúvel o alginato de sódio. É composto por grupos carboxila, sendo assim, quando desprotonado em solução, tem potencial em interagir com cátions, e estudos indicam que tal polímero pode eliminar metais pesados como $\mathrm{Pb}^{2+}, \mathrm{Cu}^{2+} \mathrm{e}$ $\mathrm{Cd}^{2+}$. Como reagente, é biodegradável e biocompatível, já que provém de algas marinhas pardas. Sendo assim, o alginato tem potencial em ser um bom agente floculante, e tem sido usado como tal de acordo com alguns estudos recentes (Rani et al., 2013; Ren et al., 2016).

A gelatina é um biopolímero com muitas aplicações industriais, como na área de alimentos, materiais e fármacos. A produção de gelatina consiste basicamente na hidrólise parcial do colágeno, sendo assim, tem uma cadeia flexível com uma alta variedade de conformações e alta presença de grupos hidroxila. Com tais características, tem uma grande interação com a água e outros compostos, representando então uma boa alternativa para floculante (Haddar et al., 2012).

O objetivo geral do trabalho foi avaliar o processo de floculação para o tratamento de uma água sintética com alto teor de sólidos por meio da avaliação comparativa do desempenho de três floculantes: alginato, quitosana e gelatina.

\section{METODOLOGIA}

\section{1 Água Sintética}

A partir de resultados dos parâmetros físico-químicos da amostra da água do Rio Doce coletada em abril/2016, a qual estava dentro dos padrões de potabilidade determinados pelo CONAMA (Brasil, 2005), decidiu-se por preparar uma solução de água sintética. Esse 
preparo foi feito de acordo com o relatório de novembro de 2015, apresentado pelo IGAM (IGAM, 2015).

Em relação à reprodução da turbidez, escolheu-se a argila como fonte de sólidos. A sua massa a ser adicionada foi determinada de acordo com o valor de sólidos suspensos totais registrados no relatório. Sobre a condutividade do meio, representada pela presença dos metais, definiu-se a quantidade de íons $\mathrm{Fe}^{2+}$ e $\mathrm{Al}^{3+}$ de acordo com os valores de concentração apresentados (IGAM, 2015).

Sendo assim, partindo dos resultados apresentados pelo IGAM, de que a concentração de alumínio, ferro, de sólidos totais na água foi de 0,22, 0,58 e $112.280 \mathrm{mg} / \mathrm{L}$ respectivamente, preparou-se a água sintética utilizando cloreto de alumínio, sulfato de ferro (II) e argila com as devidas massas. A amostra feita foi caracterizada em relação à turbidez e condutividade (APHA, 2012), que foram de $4000 \mathrm{NTU}$ e $300 \mu \mathrm{S} / \mathrm{cm}$, respectivamente.

\subsection{Floculantes}

Foram preparadas soluções dos floculantes quitosana, alginato e gelatina, com concentração de $0,5 \%$ em massa. Para a quitosana, foi utilizada como solvente uma solução aquosa de ácido acético, com concentração $5 \% \mathrm{~m} / \mathrm{v}$.

\subsection{Sedimentação em teste de bancada}

O processo de bancada de floculação foi realizado em um Jar Test (Milan, JT - 203), contendo seis cubas. Em cada cuba, foram adicionados $1,5 \mathrm{~L}$ da água sintética, e a concentração de floculante foi de 0,$003 ; 0,006 ; 0,01 ; 0,013 ; 0,016$ e $0,02 \mathrm{~g} / \mathrm{L}$ com o objetivo de definir a melhor proporção floculante/água sintética. Adicionados os reagentes, a mistura foi agitada com rotação $100 \mathrm{rpm}$ por 6 minutos e seguida de uma agitação com rotação 40 rpm por 20 minutos (Roussy, 2005).

A eficiência do floculante foi determinada de acordo com a leitura de turbidez antes e depois do processo, ou seja, com 30, 60, 120, 180 minutos e 24 horas do início da sedimentação, para estudo da cinética do fenômeno.

\section{RESULTADOS E DISCUSSÃO}

Os parâmetros avaliados para a água sintética são apresentados na Tabela 1.

Tabela 1 - Parâmetros físico-químicos da água sintética preparada

\begin{tabular}{|c|c|}
\hline Parâmetro & Valor \\
\hline Condutividade $(\mu \mathrm{S})$ & 831,7 \\
\hline Turbidez $(\mathrm{NTU})$ & 2109,42 \\
\hline Sólidos totais $(\mathrm{g} / \mathrm{L})$ & 2,2911 \\
\hline
\end{tabular}

Os resultados de turbidez em função do tempo de sedimentação para cada floculante são apresentados na Figura 1. 
Figura 1 - Evolução da turbidez em função do tempo de sedimentação usando o biofloculante: a) quitosana, b) gelatina e c) alginato

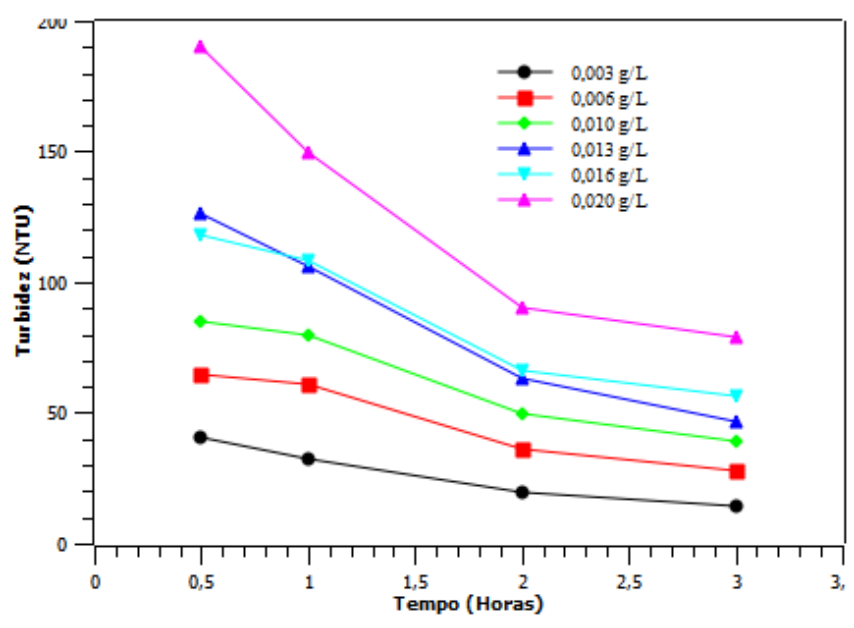

b

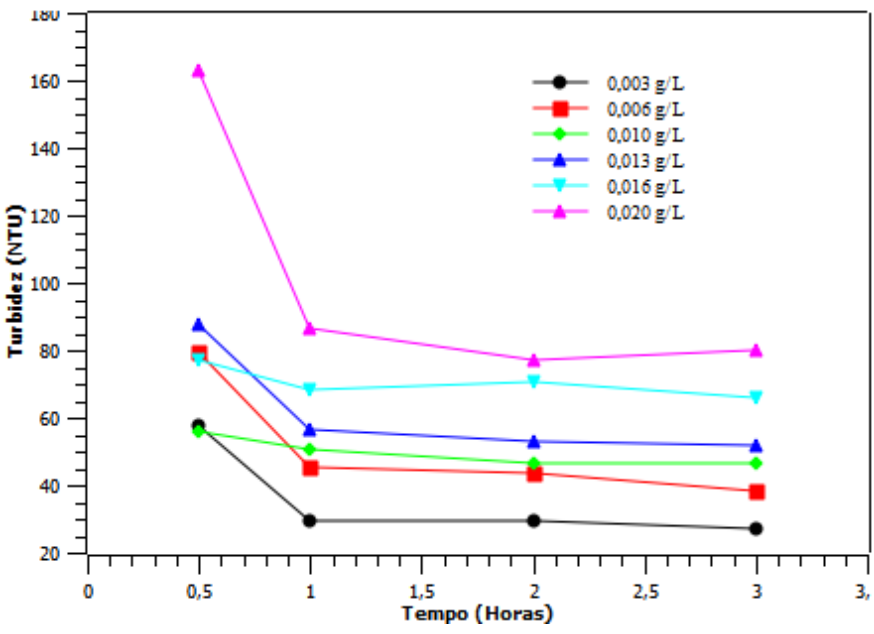

C

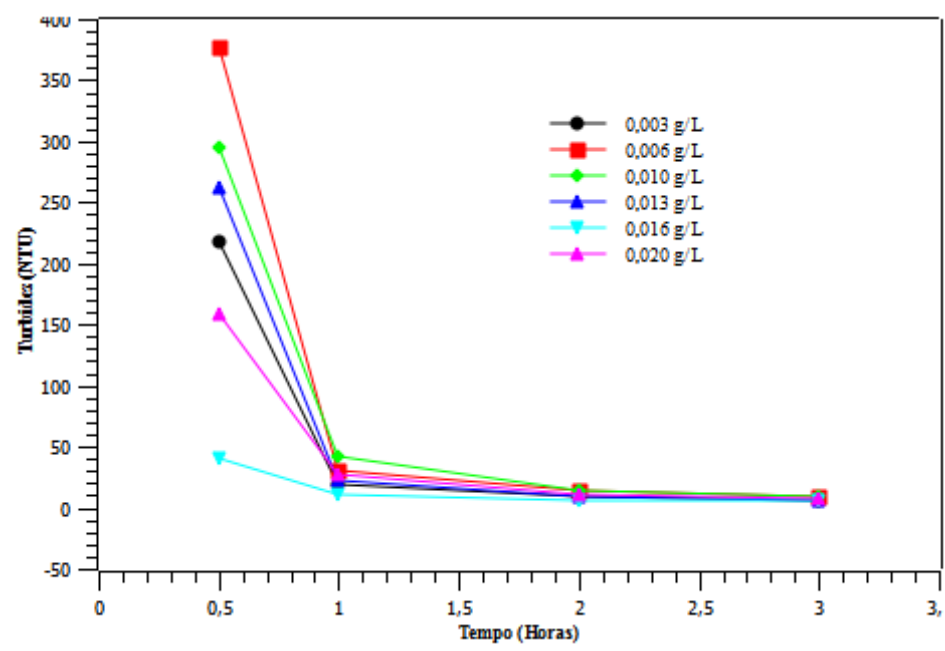

Em todos os casos, houve uma queda mais acentuada de turbidez no início do processo e, após no máximo duas horas de sedimentação, a turbidez decai de forma menos intensa. No caso do alginato, esse decaimento na turbidez foi de maior intensidade dos dois primeiros pontos para os dois últimos, quando comparado aos outros floculantes. Isso pode ter ocorrido devido à estrutura molecular desse composto e seu caráter aniônico, enquanto a quitosana e a gelatina eram catiônico e polar respectivamente. Isso fez com que, quando aglomerado durante a floculação, os flocos tenham densidade maior que os da quitosana e da gelatina. Sendo assim, sua cinética de sedimentação foi maior em relação aos demais floculantes.

Em relação à quitosana e à gelatina, percebe-se que tiveram uma uniformidade menor dos resultados. A quantidade de floculante adicionado teve maior influência no processo de sedimentação, quando comparados com o alginato. Nesse caso, pelo gráfico, é possível visualizar que, após a primeira hora de sedimentação, os valores de turbidez medidos tiveram 
uma diferença menor. Apesar disso, independente do volume adicionado de floculante, em todos os três processos separadamente, após 24 horas de sedimentação, todas as suspensões atingiram valores de turbidez de 9,90 NTU para a quitosana, 11,81 NTU para a gelatina e 0,103 NTU para o alginato.

Os melhores resultados foram obtidos usando como biofloculante a solução de alginato $0,5 \% \mathrm{~m} / \mathrm{m}$. Entretanto, não se pode deixar de afirmar que todos os três processos foram eficientes, já que a redução da turbidez da água foi em torno de $97,9 \%$ para a quitosana e a gelatina, e de praticamente $100 \%$ para o alginato, após 3 horas de sedimentação.

Em relação à dosagem de floculante, todos os casos apresentaram melhores resultados na cuba em que havia menor concentração do polímero. Isso pode ser explicado pelo fato de que, para um mesmo teor de íons, o aumento da concentração do polímero ajuda a aumentar a repulsão entre as cadeias, impedindo a floculação. Para as menores concentrações de polímeros, os íons comprimem a camada elétrica dupla e reduzem a repulsão entre as espécies, favorecendo a sedimentação.

Os ajustes dos valores de turbidez em função do tempo indicaram, para todos os sistemas, que a cinética é de segunda ordem para o processo, conforme apresentado na Tabela 2, apenas para os dados com alginato. Isso indica que o processo é bastante complexo e envolve a interação tanto da partícula sólida quanto a macromolécula.

Tabela 2 - Cinética do processo de sedimentação usando alginato como floculante.

\begin{tabular}{|l|c|c|c|c|c|c|c|c|c|}
\hline Concentração (g/L) & \multicolumn{3}{|c|}{0,003} & \multicolumn{3}{c|}{0,006} & \multicolumn{3}{c|}{0,010} \\
\hline & $\mathrm{A}$ & $\mathrm{B}$ & $\mathrm{R}^{2}$ & $\mathrm{~A}$ & $\mathrm{~B}$ & $\mathrm{r}^{2}$ & $\mathrm{~A}$ & $\mathrm{~B}$ & $\mathrm{R}^{2}$ \\
\hline Ordem 0 & $-83,035$ & 211,44 & 0,4983 & $-115,49$ & 295,22 & 0,5069 & $-92,37$ & 240,12 & 0,5554 \\
\hline $1^{\mathrm{a}}$ Ordem & $-1,32$ & 196,66 & 0,7285 & $-1,305$ & 287,18 & 0,7463 & $-1,279$ & 282,47 & 0,8301 \\
\hline $2^{\mathrm{a}}$ Ordem & 0,064 & $-0,0205$ & 0,9899 & 0,0424 & $-0,0138$ & 0,9906 & 0,0433 & $-0,0178$ & 0,9956 \\
\hline Concentração (g/L) & \multicolumn{3}{|c|}{0,013} & \multicolumn{3}{c|}{0,016} & \multicolumn{4}{c|}{0,020} \\
\hline & $\mathrm{A}$ & $\mathrm{B}$ & $\mathrm{R}^{2}$ & $\mathrm{~A}$ & $\mathrm{~B}$ & $\mathrm{R}^{2}$ & $\mathrm{~A}$ & $\mathrm{~B}$ & $\mathrm{R}^{2}$ \\
\hline Ordem 0 & $-80,428$ & 205,85 & 0,511 & $-11,881$ & 34,695 & 0,5879 & $-49,016$ & 130,43 & 0,5702 \\
\hline $1^{\text {a } \text { Ordem }}$ & $-1,303$ & 203,62 & 0,752 & $-0,766$ & 35,571 & 0,7396 & $-1,13$ & 150,8 & 0,8319 \\
\hline $2^{\mathrm{a}}$ Ordem & 0,0594 & $-0,0192$ & 0,9848 & 0,071 & 0,0137 & 0,8479 & 0,0538 & $-0,0186$ & 0,999 \\
\hline
\end{tabular}

Por fim, foi definido então que o melhor biofloculante seria o alginato $0,5 \% \mathrm{~m} / \mathrm{m}$ e que este deve ser adicionado na proporção $0,003 \mathrm{~g} / \mathrm{L}$. Rani e colaboradores (2013) caracterizaram um copolímero formado por alginato e polimetil-metacrilato e obtiveram bons resultados na floculação utilizando o copolímero em concentração 0,375 ppm.

\section{CONCLUSÃO}

$\mathrm{O}$ alginato apresentou os melhores resultados dentre os floculantes investigados, mas todos os reagentes utilizados tiveram boa eficiência, sendo essa em torno de $99 \%$. Foi determinada a concentração ótima de $0,003 \mathrm{~g} / \mathrm{L}$ do alginato na água a ser tratada., provavelmente devido às cargas negativas do alginato. Todos os floculantes investigados indicaram que o processo é regido por uma cinética de segunda ordem

\section{REFERÊNCIAS}

APHA, Standard Methods for the Examination of Water and Wastewater - 22 ed, 2012 
BRASIL. CONAMA - Conselho Nacional do Meio Ambiente. Resolução $n^{\circ}$ 357/05. Estabelece a classificação das águas doces, salobras e salinas do Território Nacional. Brasília, SEMA, 2005.

HADDAR, A.; SELlIMI, S.; GHANNOUCHI, R.; ALVAREZ, O. M.; NASRI, M.; BOUGATEF, A.; Functional, antioxidant and film-forming properties of tuna-skin gelatin with a brown algae extract. International Journal of Biological Macromolecules. v. 51, p. 477-483, 2012.

IGAM - Instituto Mineiro de Gestão das Águas. Acompanhamento da qualidade das águas do Rio Doce após o rompimento da barragem da Samarco no distrito de Bento Rodrigues Mariana/MG, 2015.

KURNIAWAN, T. A.; CHAN, G. Y. S.; LO, W.; BABEL, S. Physico-quemical treatment techniques for wastewater laden with heavy metals. Chemical Engineering Journal. v. 118, p. 83-98, 2006.

RANI, P.; MISHRA, S.; SEM, G. Microwave based synthesis of polymethyl methacrylate grafted sodium alginate: its application as flocculant. Carbohydrate Polymers. v. 91, p. 686-692, 2013.

REN, H.; GAO, Z.; WU, D.; JIANG, J.; SUN, Y.; LUO, C. Efficient Pb(II) removal using sodium alginate-carboxymethyl cellulose gel beads: Preparation, characterization, and adsorption mechanism. Carbohydrate Polymers. v. 137, p. 402-409, 2016.

ROUSSY, J.; VOOREN, M. V.; DEMPSEY, B. A.; GUIBAL, E. Influence of chitosan characteristics on the coagulation and the flocculation of bentonite suspensions. Water Research. v. 39, p. 3247-3258, 2005.

SALEHIZADEH, H.; YAN, N. Recent advances in extracellular biopolymer flocculants. Biotechnology Advances. v. 32, p. 1506-1522, 2014.

YANG, R.; LI, H.; HUANGM.; YANG, H.; LI, A. A review on the chitosan-based flocculants and their applications in water treatment. Water Research. v. 95, p. 59-89, 2016. 